\title{
ANATOMICAL VARIATIONS OF EXTRA HEPATIC BILIARY SYSTEM IN PATIENTS UNDERGOING LAPAROSCOPIC CHOLECYSTECTOMY IN CMH \& PEMH RAWALPINDI
}

\author{
Muhammad Ali Muazzam, Syed Mukarram Hussain*, Muhammad Tanveer Ahmed Qureshi** \\ Combined Military Hospital Quetta/National University of Medical Sciences (NUMS) Pakistan, *Combined Military Hospital/National University of Medical \\ Sciences (NUMS) Rawalpindi Pakistan, **Armed Forces Institute of Post Graduate Medical Institute/National University of Medical Sciences (NUMS) \\ Rawalpindi Pakistan
}

\section{ABSTRACT}

Objective: To assess the frequency of anatomical variations of the extra-hepatic biliary tract in patients undergoing laparoscopic cholecystectomy in Combined Military Hospital \& Pak Emirates Military Hospital Rawalpindi.

Study Design: Comparative cross-sectional study.

Place and Duration of Study: Department of General Surgery, Combined Military Hospital \& Pak Emirates Military Hospital, Rawalpindi, from Mar to Aug 2017.

Methodology: A total of 136 patients of either gender with cholelithiasis of more than one month were included. Participants were distributed into equal number of groups for both hospitals by lottery method. All the participants had under gone laparoscopic cholecystectomy by consultant general surgeon or senior registrar under direct supervision. Structures mainly assessed for variations were gall bladder, cystic duct, common hepatic duct, supraduodenal part of common bile duct, cystic artery, and hepatic artery which were characteristically encountered during laparoscopy.

Results: Overall Extra hepatic biliary variations were 136 (23\%), at Combined Military Hospital 68 (16\%) and Pak Emirates Military Hospital 68 (29.4\%). Gall bladder anomaly was seen in 3\% patients, cystic duct anomaly $4.4 \%$, supraduodenal part of common bile duct anomaly $0.7 \%$, cystic artery anomaly $11 \%$ and hepatic artery anomaly was seen in $3.6 \%$ patients $(p>0.05)$.

Conclusion: Anatomic variations were found to be not uncommon in our set up. Thus, there is a need for doctors to continuously refresh knowledge of normal anatomy and the variants of biliary tract.

Keywords: Anatomic variations, Extra-hepatic biliary tract, Laparoscopic cholecystectomy.

This is an Open Access article distributed under the terms of the Creative Commons Attribution License (https://creativecommons.org/licenses/by-nc/4.0/), which permits unrestricted use, distribution, and reproduction in any medium, provided the original work is properly cited.

\section{INTRODUCTION}

Cholelithiasis is a worldwide surgical problem with anatomical, geographical, racial and ethnic variations ${ }^{1}$. Alexander trallianus first described gall stones within bile duct and it was first reported by Fallopius and Vesalius in gallbladder by 16th century during dissection ${ }^{2}$.

Globally its incidence is $10-20 \%$ in adult population. In Asian countries its prevalence ranges from 3\% to $10 \% 1,3$.

Its treatment is only surgical which is cholecystectomy. First cholecystectomy was performed in 1882 by Langenbuch ${ }^{4}$ by a large right subcostal incision. Humanity had to wait for a decade for smaller incision cholecystectomy i.e., laparoscopic. First cholecystectomy via laparoscopy was performed in $1987^{5}$.

Laparoscopic cholecystectomy (LC) has supplanted open cholecystectomy and now is the operation of choice for biliary tractailments. More than $90 \%$ elective and $70 \%$ of emergency cholecystectomy is carried out via laparoscopic approach making LC as one of the

Correspondence: Dr Muhammad Ali Muazzam, Dept of Surgery, Combined Military Hospital, Quetta Pakistan

Received: 24 Sep 2019; revised received: 17 Dec 2019; accepted: 23 Dec 2019 frequently perform surgery globally ${ }^{6}$.

Biliary injury is a commonly encountered complication after LC, increases to $0.8 \%$ with the advent of laparoscopy while open cholecystectomy remains $0.2-$ $0.3 \%$ mostly from inability to outline Calot's triangle anatomy. Typical biliary tract anatomy is found in only $20-40 \%$ of cases ${ }^{7}$.

Extra hepatic biliary tract (EHBT) comprises of four parts. Cystic duct and gall bladder, right and left hepatic ducts, common hepatic and bile duct and the pancreatic and intraduodenal parts 8 .

Variation in the anatomy of EHBT is significant, as failure to identify these will lead to accidentalduct ligation, biliary leaks and stricture after LC 8 -10.

LC is a routine procedure in our setup for cholelithiasis which involves a standard four ports access with two $5 \mathrm{~mm}$ right side ports, $12 \mathrm{~mm}$ epigastric port whereas umbilical port remains a surgeon choice either $5 \mathrm{~mm}$ or $12 \mathrm{~mm}$.

The present study was intended to throwlight on different anatomical variations of EHBT in patients of Combined Military Hospital (CMH) Rawalpindi and Pak Emirates Military Hospital (PEMH). Knowledge of anatomy of EHBT as well as their variants issignificant. 
Inability to identify them and their various variations in extra hepatic biliary system may result in accidental duct ligation, biliary leakage and strictures after LC, which may cause aftermath drastic suffer to patient as well as markedly reduce the cost effectiveness of laparoscopic surgery and surgeon in public. With these results in mind the operating surgeons will be more meticulous while performing LC.

\section{METHODOLOGY}

This comparative cross-sectional study was carried out at department of General Surgery of Combined Military Hospital and Pak Emirates Military Hospital Rawalpindi. Sample size of 136 was calculated using Open Epi version 2.3 Software with following assumptions confidence interval; $95 \%$ anatomical variations present: 9.6\%12. Desired precision: 5\% Total sample size (n): 136.

After approval from ethical review board, a total of 136 patients of both gender and age limits $>18$ years and $<65$ years with cholelithiasis of $>1$ month with informed consent were included in the study. Participants with ASA III \& IV, empyema gallbladder, acute pancreatitis, obstructive jaundice and carcinoma gallbladder were excluded.

All eligible patients fulfilling inclusion criteria admitted through outpatient department (OPD) after completing full workup for surgery and anesthetic fitness. In the morning before operation, participants took bath. Surgical site was made ready under aseptic standard protocol.

All the patients undergone laparoscopic cholecystectomy by a consultant general surgeon or senior registrar as per both hospital protocols having $>2$ years of postfellowship experience. Gall bladder, cystic duct, common hepatic duct, supraduodenal part of common bile duct, cystic artery, and hepatic artery were assessed for variations. However, assessment of variations of hepatic ducts, portal vein, retro duodenal and pancreatic parts of CBD was not be possible because of iatrogenic injuries.

Data entered and analyzed using SPSS-23. Mean \pm SD computed for quantitative variable. Frequency and percentage were calculated for all the qualitative variables. Chi-square test was used for assessment of Statistically significant. A $p$-value $<0.05$ considered significant.

\section{RESULTS}

A total of 136 patients were enrolled in this study. Mean Age was $39.592 \pm 7.78$ years. Majority of the patients were females 136 (79.4\%). More patients belonged to ASA-I score 136 (70.5\%). Variations of extra hepatic biliary tract were seen in $31(23 \%)$ patients.

Gall bladder anomaly was seen in 3\% patients, cystic duct anomaly $4.4 \%$, supraduodenal part of common bile duct anomaly $0.7 \%$, cystic artery anomaly $11 \%$ and hepatic artery anomaly was seen in $3.6 \%$ patients as shown in table.

Table: Clinical characteristics of the patients.

\begin{tabular}{|c|c|c|c|}
\hline \multirow{2}{*}{ Factors } & \multicolumn{2}{|c|}{ Study Parameter } & \multirow{2}{*}{$p$-value } \\
\hline & Yes & No & \\
\hline \multicolumn{4}{|c|}{ Variation of Extrahepatic Biliary Tract } \\
\hline $\mathrm{CMH}$ & $7(25)$ & $21(75)$ & \multirow{3}{*}{0.773} \\
\hline PEMH & $24(22.2)$ & $84(77.7)$ & \\
\hline Total & $31(23)$ & $105(77)$ & \\
\hline \multicolumn{4}{|c|}{ Gall Bladder Variation } \\
\hline $\mathrm{CMH}$ & $1(3.6)$ & $27(96.4)$ & \multirow{3}{*}{0.831} \\
\hline PEMH & $3(2.8)$ & $105(97.2)$ & \\
\hline Total & $4(3)$ & $132(97)$ & \\
\hline \multicolumn{4}{|c|}{ Cystic Duct Variation } \\
\hline $\mathrm{CMH}$ & $2(7.1)$ & $26(92.9)$ & \multirow{3}{*}{0.436} \\
\hline PEMH & $4(85.1)$ & $104(96.3)$ & \\
\hline Total & $6(4.4)$ & $130(95.6)$ & \\
\hline \multicolumn{4}{|c|}{ Supra-duodenal Part of Common Bile Duct Variations } \\
\hline $\mathrm{CMH}$ & - & $28(100)$ & \multirow{3}{*}{0.608} \\
\hline PEMH & $1(0.9)$ & $107(99.1)$ & \\
\hline Total & $1(0.7)$ & $135(99.3)$ & \\
\hline \multicolumn{4}{|c|}{ Cystic Artery Variations } \\
\hline $\mathrm{CMH}$ & $4(14.3)$ & $24(85.7)$ & \multirow{3}{*}{0.548} \\
\hline PEMH & $11(10.2)$ & $97(89.8)$ & \\
\hline Total & $15(11)$ & $121(88.9)$ & \\
\hline \multicolumn{4}{|c|}{ Hepatic Artery Variations } \\
\hline $\mathrm{CMH}$ & - & $28(100)$ & \multirow{3}{*}{0.244} \\
\hline PEMH & $5(4.6)$ & $103(95.3)$ & \\
\hline Total & $5(3.7)$ & $131(96.3)$ & \\
\hline
\end{tabular}

\section{DISCUSSION}

Laparoscopy allows exploration of biliary tract anatomy owing to high resolution and magnification. Therefore, extrahepatic biliary system can certainly be evaluated for its anatomical variants and congenital abnormalities during LC.

Talpur et al in 2010 reported that anatomical variations were observed in $20.3 \%$ patients. Of these, $52.5 \%$ had cystic artery anomalies, $21.3 \%$ had cystic duct anomalies, $13.1 \%$ had right hepatic artery anomalies, 9.8\% had gall bladder anomalies and 3.3\% had common hepatic artery ${ }^{9}$.

Awazli et al in 2013 reported incidence of EHBT as $54 \%$. Furthermore, this study reported that, these extra-hepatic biliary tract cases included vascular anomalies (40\%); ductal anomalies (12\%); gallbladder ano- 
malies (2\%); mostly occurred as Phrygian cap (1.3\%). Anatomical variations were observed more in females as compared to males $(80 \% \text { vs } 20 \%)^{10,11}$.

Dawani et al in 2013 reported that anatomical variations were observed in $13(9.6 \%)$ patients only. In these patients Moynihan's hump was seen in 8 (5.9\%) whereas accessory cystic artery was noted in 5 (3.7\%) patients ${ }^{11}$.

Khayat et al in 2014 reported prevalence of abnormal anatomy in extra-hepatic biliary tract as $20 \% 12$.

Hasan et al in 2014 revealed variations in extrahepatic biliary tract of $15.2 \% 13$.

Devi et al in 2014 shows extra hepatic biliary apparatus variations as $20 \% 14$.

Khan et al in 2008 reported anatomical variants in $14 \%$ surgeries, among stirregularities of 14 cases, Moynihan's hump in 6\%, accessory cystic artery in 6\%, Double cystic duct in 1\% and long cystic duct in $1 \%$ of the cases ${ }^{15}$.

Literature suggest soccurrence of accessory cystic duct in $1-30 \%$ of cases ${ }^{16,17}$.

Singh et al in 2017 reported in study $26.6 \%$ vascular anomalies and $12.16 \%$ ductal anomalies ${ }^{18}$.

Farooq et al in 2019 noted variations in cystic artery during laparoscopic were it was single (92.25\%), originating from right hepatic artery $(90.25 \%)$, crossing cystic duct anteriorly $(72.75 \%)$ and of $2-3 \mathrm{~cm}$ in length $(68 \%)^{19}$.

Rodrigues et al in 2019 described in his study that the anatomy of the biliary tree is complex, and its variations of both intra- and extra-hepatic bile ducts can be found in approximately 30\% of the general population $^{20}$. Sen et al in 2020 reported that the incidence of biliary anomalies varies from $15-66 \% 21$.

Naeem et al in 2020 described that standard anatomy of EHBT was found to be prevalent in $65.8 \% 22$.

In this study overall extrahepatic biliary variations were $23 \%$, at CMH $16 \%$ and PEMH $29.4 \%$. Gall bladder anomaly was seen in 3\% patients, cystic duct anomaly $4.4 \%$, supraduodenal part of common bile duct anomaly $0.7 \%$, cystic artery anomaly $11 \%$ and hepatic artery anomaly was seen in $3.6 \%$ patients. No operative complications occur in all study patients during procedure neither any conversion to open cholecystectomy occur.

\section{CONCLUSION}

Anatomic variations were found to be not uncommon in our set up. These anatomical variations were susceptible to injuries during cholecystectomy, thus there is a need for doctors to continuously refresh knowledge of normal anatomy and the variants of biliary tract.

\section{CONFLICT OF INTEREST}

This study has no conflict of interest to be declared by any author.

\section{REFERENCES}

1. Njeze GE. Gallstones. Niger J Surg 2013; 19(2): 49-55.

2. Ahmed A, Ranjan SK, Sinha DK, Kerketta MD, Usha P. Changing incidence of gall stone disease: a single centre study from eastern India. IOSR J Dent Med Sci 2015; 14(12): 50-53.

3. Chen $\mathrm{CH}$, Huang $\mathrm{MH}$, Yang JC. Prevalence and risk factors of gallstone disease in an adult population of Taiwan: an epidemiological survey. J Gastr Hepatol 2006; 21(2): 1737-43.

4. Sparkman RS. $100^{\text {th }}$ Anniversary of the First Cholecystectomy: A Reprinting of the $50^{\text {th }}$ Anniversary Article From the Archives of Surgery, July 1932. Arch Surg 1982; 117(12): 1525.

5. Testas P, Dewatteville JC. Laparoscopic cholecystectomy. Ann Gastroenterol Hepatol (Paris) 1993; 29(6): 300-06.

6. Sheffield KM, Ramos KE, Djukom CD, Jimenez CJ, Mileski WJ, Kimbrough TD, et al. Implementation of a critical pathway for complicated gallstone disease: translation of population-based data into clinical practice. J Am Coll Surg 2011; 212(5): 835-43.

7. Wu YV, Linehan DC. Bile duct injuries in the era of laparoscopic cholecystectomies. Surg Clin North Am 2010; 90(4): 787-802.

8. Anupama D, Shivaleela C, Lakshmiprabha S. A study of Anatomy of extrahepatic ducts and its variations with clinical significance. Int J Anatomy Res 2016; 4(1): 2029-33.

9. Talpur KA, Laghari AA, Yousfani SA, Malik AM, Memon AI, Khan SA. Anatomical variations and congenital anomalies of extra hepatic biliary system encountered during laparoscopic cholecystectomy. J Pak Med Assoc 2010; 60(2): 89-93.

10. Awazli LG. Anatomical variations of extrahepatic biliary system. Iraqi J Med Sci 2013; 11(3): 258-64.

11. Dawani S, Sandhya A, Rasul S. Frequency of common anatomical variations in the extrahepatic biliary tract in patients undergoing elective cholecystectomy. Pak J Surg 2013; 29(1): 61-5.

12. Khayat MF, Al-Amoodi MS, Aldaqal SM, Sibiany A. Abnormal anatomical variations of extra-hepatic biliary tract, and their relation to biliary tract injuries and stones formation. Gastroenterol Res 2014; 7(1): 12-16.

13. Hasan MM, Reza E, Khan MR, Laila SZ, Rahman F, Mamun MH. Anatomical and congenital anomalies of extrahepatic biliary system encountered during cholecystectomy. Mymensingh Med J 2014; 22(1): 20-26.

14. Devi T, Krishna P. The study of variations of Extrahepatic Biliary Apparatus. J Med Dent Sci 2014; 5(5): 25-31.

15. Khan AH, Zaheer M. Frequency of extra hepatic biliary tree anomalies seen during cholecystectomy. Ann Pak Inst Med Sci 2008; 4(4): 198-200.

16. Hashimoto M, Ishikawa T, Lizuka T, Matsuda M, Watanabe G. Right hepatic duct emptying into the cystic duct: Report of aCase. Surg Endosc 2002; 16(2): 359.

17. Losanoff JE, Jones JW, Richman BW, Rangnekar NJ. Hepaticocystic duct: A rare anomaly of the extrahepatic biliary system. Clin Anat 2002; 15(4): 314-15.

18. Singh K, Singh R, Kaur M. Clinical reappraisal of vasculobiliary anatomy relevant to laparoscopic cholecystectomy. J Minim Access Surg 2017; 13(4): 273-79. 
19. Farooq S, Jahan N, Arshad S. Anatomical variations of cystic artery during laparoscopic cholecystectomy; an audit of 400 cases oflaparoscopic surgery for gall bladder pathologies at a tertiary care unit. Ann Punjab Med Coll 2019; 13(1): 72-75.

20. Rodrigues G, Pandit SR, Khan A, Veerabharappa B, Jayasankar B, Anaparti R. High insertion of cystic duct at the gallbladder fundus: An undescribed anomaly. J Minim Access Surg 2019; 15(3): 256-58.
21. Sen S, Goel S, Gaur M. Anatomical variation of extra-hepatic biliary tree and vasculature encountered during laparoscopic cholecystectomy. Intl J Contemporary Surg 2020; 8(2): 20-25.

22. Naeem MQ, Ahmed MS, Hamid K, Shazlee MK, Qureshi F, Asad Ullah M. Prevalence of different hepatobiliary tree variants on magnetic resonance cholangiopancreatography in patients visiting a tertiary care teaching hospital in Karachi. Cureus 2020; 12(12): 1-3. 